\title{
Stage IIIA Adult Liver Cancer AJCC v7
}

National Cancer Institute

\section{Source}

National Cancer Institute. Stage IIIA Adult Liver Cancer A/CC v7. NCI Thesaurus. Code C94779.

For hepatocellular carcinoma: Stage IIIA includes: T3a, NO, M0. T3a: Multiple tumors more than $5 \mathrm{~cm}$. N0: No regional lymph node metastasis. M0: No distant metastasis. (AJCC 7th ed.) 